\title{
Pyomyositis with Iliac Vein Thrombophlebitis and Septic Pulmonary Embolism: A Unique Case of Reverse Lemierre Syndrome
}

\author{
Vishwanath Vijay Joshi, Mitusha Verma, Neemish Kamat, Deepak Patkar \\ Department of Radiology, Dr Balabhai Nanavati Hospital, Mumbai-400056, Maharashtra, India.
}

\section{Corresponding Author:}

Dr Vishwanath Vijay Joshi

Email: vishuvjoshi@gmail.com

This is an Open Access article distributed under the terms of the Creative Commons Attribution License (creativecommons.org/ licenses/by/3.0).

Received : October 29,2020

Accepted : October 5, 2021

Published : October 20,2021

\begin{abstract}
Background: The reverse Lemierre's syndrome refers to a septic thrombosis in noncervical sites. Case Report: A 40 years old diabetic patient presented with fever and acute onset pain in left gluteal region radiating to left thigh. Magnetic resonance imaging (MRI) showed pyomyositis and internal iliac vein thrombosis. Computed tomography (CT) venography showed non-enhancing filling defects within common iliac propagating into internal iliac veins along with intramuscular abscesses while whole body positron emission tomography (PET-CT) revealed bilateral scattered pulmonary nodules suggestive of septic emboli. The patient responded to broad spectrum antibiotics. Conclusion: This unique case of reverse Lemierre syndrome highlights the importance of multimodality imaging including PET-CT in identifying additional foci infection in lungs.
\end{abstract}

Keywords: Diabetes Mellitus, Lemierre's Syndrome, Magnetic Resonance Imaging, Postanginal Sepsis. Thrombophlebitis.

\section{Introduction}

Primary pyomyositis or spontaneous bacterial myositis is characterized by suppuration of single or multiple skeletal muscles [1,2]. Septic pulmonary embolism (SPE) occurs when emboli containing pathogens traverse via pulmonary artery to cause pulmonary embolism and focal lung abscesses with high morbidity and mortality $[3,4]$. Rarely both entities co-exist with each other. Here we report a unique case of pyomyositis complicated by septic thrombophlebitis of iliac veins and resulting in septic pulmonary emboli and necrotizing pneumonia, thus resembling a case of reverse Lemierre syndrome.

\section{Case Report}

A 40-year-old man presented to emergency department with fever since 3 days and left thigh and hip pain since 10 days. The pain was partially relieved with analgesics. The patient was a known case of type II diabetes mellitus on oral hypoglycemics since 4 years. General examination revealed anemic patient having tachypnea with a respiratory rate of 38 per minute, tachycardia of 122 per minute and normal blood pressure of 114/86 $\mathrm{mm}$ of $\mathrm{Hg}$. Local examination showed tender swelling of the left gluteal and proximal thigh regions with restricted movements. Respiratory examination revealed bilateral fine crepts in the infra-scapular areas. Rest of the systemic examination including abdomen, cardiovascular and the central nervous systems were normal.

Routine blood investigations showed microcytic hypochromic anemia, leukocytosis with neutrophilia. All the inflammatory markers were raised viz ESR: $50 \mathrm{~mm} / 1^{\text {st }} \mathrm{hr}$, C-reactive protein: $150 \mathrm{mg} / \mathrm{L}$ and ferritin $650 \mathrm{ng} / \mathrm{mL}$. The patient's random blood sugar was $360 \mathrm{mg} / \mathrm{dL}$ with HbA1c of $8.1 \%$ indicating poor glycemic control. The chest radiograph revealed right lower zone infiltrates with blunting of costo-phrenic angle suggestive of likely pleural effusion. Based on the above clinical findings, provisional diagnosis 
of cellulitis with septicemia and uncontrolled diabetes mellitus was made and patient was puton broad-spectrum antibiotics. After 2 days of admission, the pain in gluteal region increased and patient was referred for MRI of left gluteal region to rule out any collections or foci of infection. MRI showed diffuse hyper-intensity on T2 weighted images suggestive of edema within the pyriformis, left gluteal muscles, obturator and proximal adductor group of thigh [Fig.1a]. T2 hyper-intense collections were seen in pyriformis and left gluteus medius muscles suggestive of likely abscesses [Fig.1b]. A feature of note in the MRI study was absence of flow void in left common iliac and internal iliac veins [Fig.2a,b] suggestive of likely deep venous thrombosis which was confirmed later on ultrasound examination. In order to assess the extent of venous thrombosis, CT venography of abdomen and bilateral lower limbs was done. The IVC, right common, internal and external iliac veins were well opacified. An expansile hypo-attenuating non-enhancing occlusive filling defect suggestive of a thrombus was seen in the left common iliac vein propagating into the left internal and external iliac veins [Fig.3a,b] with patchy opacification of the deep venous system of the left lower limb. Diffuse subcutaneous edema of the left lower limb secondary to the thrombotic occlusion [Fig.3c] and peripherally enhancing collections were seen within left gluteal and pyriformis muscles [Fig.3d]. The patient was then started on anti-thrombotic therapy along with antibiotics. On fourth day, the patient developed breathlessness with increasing spikes of fever. In order to rule out additional foci of infection elsewhere in the body, whole body fluoro-deoxy glucose (FDG) PET-CT was done.

In the lungs, PET-CT findings were remarkable for discrete nodules in the sub-pleural location of bilateral upper lobes and in right lower lobe with SUV MAX of 1.2 to 1.5 [Fig.4a]. The peripherally enhancing collections in left gluteal region and pyriformis muscles were FDG avid with SUV MAX of 11.9 [Fig.4b]. No obvious erosion or

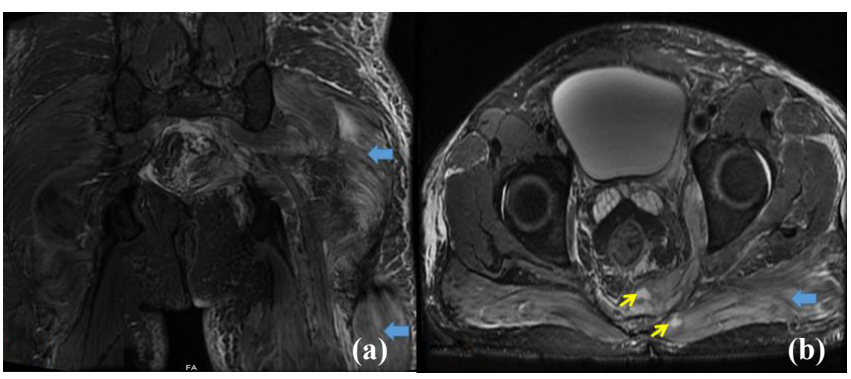

Fig.1 (a,b): Coronal and axial T2 weighted images showing diffuse edema of left gluteal and proximal thigh muscles (thick arrows). Multiple T2 hyper-intensities seen within the pyriformis (yellow arrows) and gluteus medius muscle.

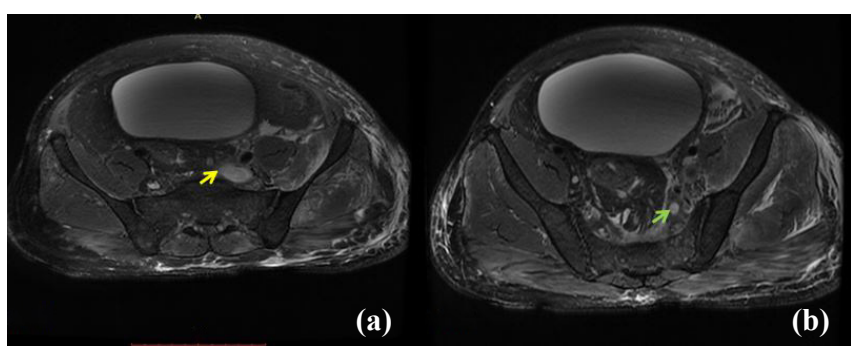

Fig.2: Fat saturated T2 images showing loss of flow void in (a): left common iliac and (b): internal iliac veins.

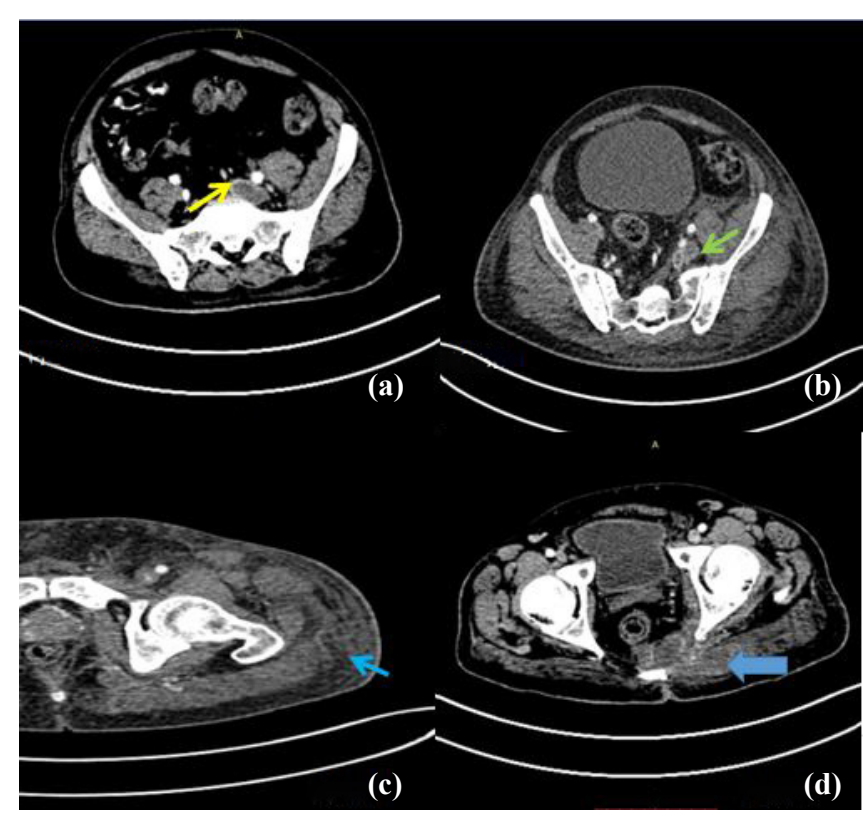

Fig.3 (a-d): Axial CT venography showing non-enhancing hypo-attenuating filling defects in left common iliac (yellow arrow) and internal iliac veins (green arrows). Diffuse edema seen around left hip (blue arrow) with peripherally enhancing collection in left gluteal muscle (thick arrow) suggestive of pyomyositis. 
sclerosis of the sacrum was seen. No uptake was seen within the thrombosed left iliac veins [Fig.4c]. Few of these show central umbilication/cavitation within suggestive of septic pulmonary emboli [Fig.5a,b]. There was also an area of air space consolidation in left lower lobe areas of parenchymal breakdown [Fig.5c] showing an SUV MAX of 3.6 suggestive of necrotizing pneumonia. There were also isolated metabolically active lymph-nodes in mediastinum probably suggestive of infective etiology. Mild bilateral pleural effusions were seen more so on left [Fig.5d] with no FDG uptake/ pleural enhancement or thickening. There were no metabolically active focal lesions in the liver, spleen or in the adrenal glands. The blood culture reports were positive for Staphyloccus aureus sensitive to both linezolid and meropenem. The patient was put on combination of these antibiotics for 4 weeks with good treatment response. Subsequently his clinical and his blood culture turned negative after one month. Currently the patient is on follow up with anti-platelets and anti-diabetic medications.

This case is unique in which it illustrates what initially appeared to be primary pyomyositis in this case of uncontrolled diabetes subsequently turned out to have septic iliac vein thrombosis and pulmonary embolism with necrotizing pneumonia on imaging workup, thus a rare case of reverse Leimerre syndrome.

\section{Discussion}

Pyomyositis can be either primary when it results from hematogenous spread or secondary when it results from contiguous spread of infection from the soft tissue or penetrating injuries [3]. Primary pyomyositis results from hematogeneous spread of infection with Staphylococcus aureus being the most common organism. The most common location of pyomyositis is involvement of the quadriceps followed by the gluteal muscles, but it can involve any skeletal muscle [5]. About 25\% of patients will have multiple abscesses within one muscle [6] whereas multiple muscle involvement

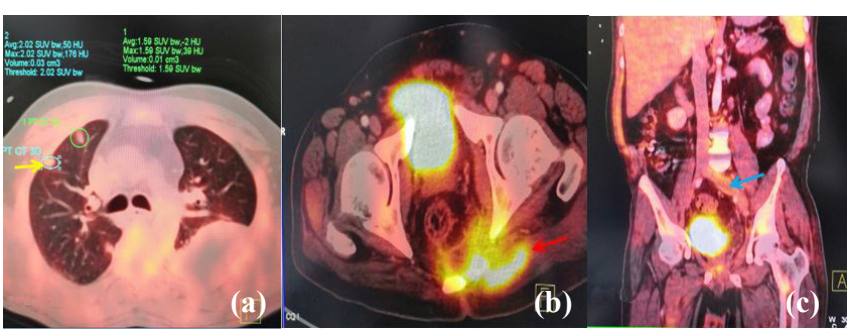

Fig.4 (a,b): Axial CT images in lung window showing subpleural nodules (yellow arrows) in both upper lobes with central umbilication/cavitation; (c): area of air space consolidation in left lower lobe with areas of parenchymal breakdown (blue arrow) suggestive of necrotizing pneumonia; (d): bilateral pleural effusions (red arrows) with subpleural nodule in right lower lobe.

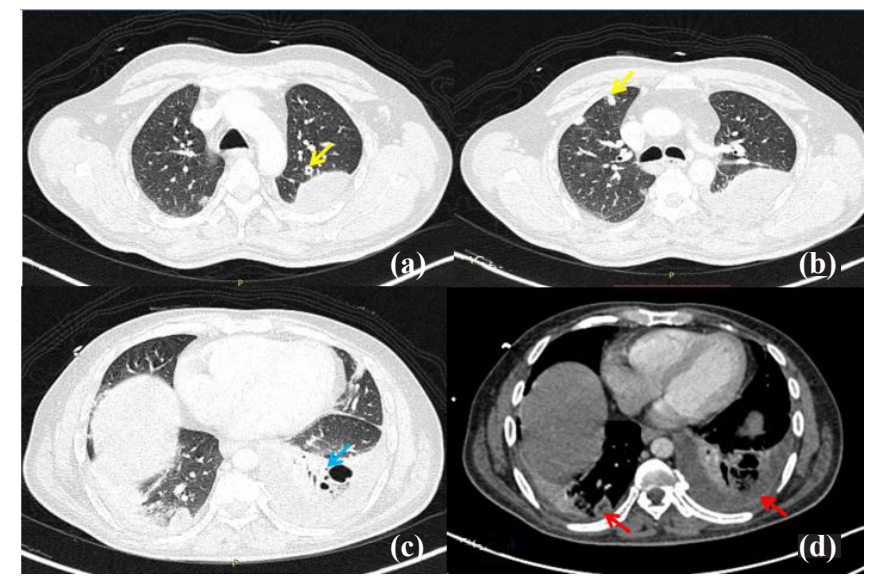

Fig.5 (a,b): Fused PET-CT images showing low metabolic activity of 2.0 in subpleural lung nodules (yellow arrows) along with intense FDG uptake in left gluteal and pyriformis muscles (red arrow); (c): thrombus within left common iliac vein with no uptake on PET-CT.

is seen in $12-40 \%$ of cases. Primary pyomyositis usually occurs in diabetics and immunosuppressed individuals. Our patient was also diabetic with uncontrolled sugar levels and involving more than muscle.

Clinically, pyomyositis can divided into a subacute phase (between 1 to 3 weeks) characterized by cramping muscle pain associated with low-grade fever and a "suppurative stage" (at 4-7 weeks) in which patient commonly presents with frank muscle tenderness and fever and local fluctuance due to intramuscular abscess formation 
$[5,6]$. The diagnosis of infectious myositis is suspected by clinical and laboratory findings, but confident diagnosis relies on imaging identification of muscle inflammation and abscess [7].

Ultrasonography shows diffuse swelling and hyper-echogenicity of the muscle due to edema with associated hypo-echoic collections in later stages representing intramuscular abscesses [8]. $\mathrm{CT}$ and MRI are complementary to ultrasound and better evaluate deeper muscle tissues and help to exclude differential diagnosis such as osteomyelitis and septic arthritis [9]. CT findings in pyomyositis include swelling and decreased attenuation of the affected muscle with effacement of surrounding fat planes. Intramuscular fluid collections within may represent areas of necrosis or abscess formation [10]. Similarly, MRI in early stages typically depicts muscle enlargement and intramuscular edema, which presents with high T2W/STIR signal and a slightly higher signal on T1W compared to uninvolved muscles [11]. Post-contrast T1W may demonstrate diffuse contrast enhancement of the affected muscle. Administration of contrast media will show rim enhancement in abscesses and also useful in differentiating enhancing viable muscle from non-enhancing necrotic tissue [12].

In our patient, diffuse intramuscular edema was seen with T2 hyper-intensities within showing peripheral enhancement suggestive of abscesses. In our case in addition to the diagnosis of pyomyositis, patient also had deep venous thrombosis of left common iliac and internal iliac veins extending to external iliac vein. This raised the suspicion of septic thrombophlebitis. PET-CT was done in view of increasing spikes of fever and breathlessness showed discrete nodules in the sub-pleural location of bilateral upper lobes and in right lower lobe with central umbilication/cavitation suggestive of septic pulmonary emboli. Additionally, an area of air space consolidation in left lower lobe with parenchymal breakdown suggestive of necrotizing pneumonia was seen. The classic Lemierre's syndrome refers to a septic thrombosis of the internal jugular vein, usually caused by a Fusobacterium necrophorum infection starting in the oral cavity, and typically complicated by pulmonary emboli [13]. The occurrence of thrombosis in non-cervical sites has been described as atypical or reverse Lemierre's syndrome. Lemierre's syndrome almost invariably arises in patients who were previously fit. This is in striking contrast to necrobacillosis arising outside the head and neck have underlying predisposing factor like cancer, diabetes mellitus or alcohol or drug abuse [14].

The basic pathophysiology of all septic thrombophlebitis are similar regardless of the vein involved. All components of Virchow's triad i.e endothelial dysfunction (contributed by inflammatory mediators and microbial toxins), stasis of blood (by compression of vein), platelet aggregation and hypercoagulable state (by sepsis) contribute to thrombus formation. This also acts as a source of septic emboli and the most common site is lung resulting in septic emboli, pulmonary abscesses and empyema (85\%), pneumatoceles, pneumothorax and acute respiratory distress syndrome $(10 \%) \quad[14,15]$. Imaging plays an indispensable role in the diagnosis of Lemierre's syndrome with use of initial ultrasonography of neck to demonstrate internal jugular vein thrombosis followed by CT or MRI to accurately delineate the extent of thrombosis and also to identify any abscesses. Septic emboli in the lungs have a characteristic radiographic appearance of multiple peripheral round and wedge shaped opacities that rapidly progress to cavitation [16]. On contrast CT "feeding vessel sign" if present is characteristic of septic pulmonary embolism. Thus, final diagnosis in our patient with uncontrolled diabetes mellitus was primary pyomyositis complicated by suppurative iliac vein thrombophebitis and thrombosis with septic pulmonary emboli and necrotizing pneumonia likely reverse Lemierre syndrome.

A search of literature showed similar case reports by Mathew et al. [17] and Talupula et al. 
[18] which also highlighted the need for prompt diagnosis of associated septic thrombophlebitis and pulmonary embolism in pyomyositis cases for good treatment outcomes. Treatment in such cases consists of supportive therapy with intravenous antibiotics, anti-coagulation along with surgical debridement/drainage. Our case showed good response to antibiotics obviating the need for surgical intervention.

\section{Conclusion}

This case reports highlights the importance of use of multimodality imaging including PET-CT in making an accurate diagnosis of reverse Lemierre's syndrome. High clinical suspicion is required in cases of primary pyomyositis to rule out associated septic thrombophlebitis and pulmonary embolism thereby reducing significant morbidity and mortality.

Contributors: VVJ: case details, manuscript preparation; MV: manuscript preparation and editing; NK: manuscript editing; DP: concept and manuscript editing. VVJ will act as a study guarantor. All authors approved the final version of this manuscript and are responsible for all aspects of this study.

Funding: None; Competing interests: None stated.

\section{References}

1. Chauhan S, Jain S, Varma S, Chauhan SS. Tropical pyomyositis (myositis tropicans): current perspective. Postgrad Med J. 2004;80:267-270.

2. Gambhir IS, Singh DS, Gupta SS, Gupta PR, Kumar M. Tropical pyomyositis in India: a clinico-histopathological study. J Trop Med Hyg. 1992;95:42-46.

3. Crum-Cianflone NF. Bacterial, fungal, parasitic, and viral myositis. Clin Microbiol Rev. 2018;21:473-494.

4. Ye R, Zhao L, Wang C, Wu X, Yan H. Clinical characteristics of septic pulmonary embolism in adults:
A systematic review. Respiratory Medicine. 2014;108: $1-8$.

5. Chiedozi LC. Pyomyositis. Review of 205 cases in 112 patients. Am J Surg. 1979;137:255-259.

6. Shepherd JJ. Tropical myositis: is it an entity and what is its cause. Lancet. 1983;322:1240-1242.

7. Chau CLF, Griffith JF. Musculoskeletal infections: Ultrasound appearances. Clin Radiol. 2005;60:149-159.

8. Trusen A, Beissert M, Schultz G, Chittka B, Darge K. Ultrasound and MRI features of pyomyositis in children. Eur Radiol. 2003;13:1050-1055.

9. Altmayer S, Verma N, MD, Dicks EA, Oliveira A. Imaging musculoskeletal soft tissue infections. Semin Ultrasound CT MRI. 2020;41:85-98.

10. Struk DW, Munk PL, Lee MJ, Ho SG, Worsley DF. Imaging of soft tissue infections. Radiol Clin North Am. 2001;39:277-303.

11. Subhawong TK, Jacobs MA, Fayad LM. Diffusionweighted MR Imaging for characterizing musculoskeletal lesions. Radio Graphics. 2014;34:1163-1177.

12. Laurencet ME, Zufferey SR, Schrenzel J. Atypical presentation of Lemierre's syndrome: case report and literature review. BMC Infectious Diseases. 2019;19:868.

13. Hagelskjaer LH, Prag J, Malczynski J, Kristensen JH. Incidence and clinical epidemiology of necrobacillosis, including Lemierre's syndrome, in Denmark 1990-1995. Eur J Clin Microbiol Infect Dis. 1998;17:561-565.

14. Alherabi A. Case of Lemierre syndrome. Ann Saudi Med. 2009;29:58-60.

15. Smith SA. Respiratory failure as a complication of pharyngitis: Lemierre's syndrome. Pediatric Emerg Care. 1999;15:402-403.

16. Screaton NJ, Ravenel JG, Lehner PJ, Heitzman ER, Flower CD. Lemierre's syndrome: forgotten but not extinct-report on four cases. Radiology. 1999;213:369374.

17. Mathew UE, Ray A, Soneja M, Vyas S, Shanker K, Nischal $\mathrm{N}$, et al. Pyomyositis complicated by deep venous thrombosis - A unique case of reverse Lemierre's syndrome. J Assoc Physicians India. 2017;65:87-91.

18. Talupula RM, Raya A, Manchanda S, Sood R. Reverse Lemierre syndrome: a case report. Egyptian Journal of Bronchology. 2018;12:363-366. 